\title{
EXACT DISTRIBUTIONS FOR REWARD FUNCTIONS ON SEMI-MARKOV AND MARKOV ADDITIVE PROCESSES
}

\author{
VALERI T. STEFANOV,${ }^{*}$ The University of Western Australia
}

\begin{abstract}
The distribution theory for reward functions on semi-Markov processes has been of interest since the early 1960s. The relevant asymptotic distribution theory has been satisfactorily developed. On the other hand, it has been noticed that it is difficult to find exact distribution results which lead to the effective computation of such distributions. Note that there is no satisfactory exact distribution result for rewards accumulated over deterministic time intervals $[0, t]$, even in the special case of continuous-time Markov chains. The present paper provides neat general results which lead to explicit closedform expressions for the relevant Laplace transforms of general reward functions on semi-Markov and Markov additive processes.
\end{abstract}

Keywords: Semi-Markov process; reward function; Laplace transform; stopping time; exponential family

2000 Mathematics Subject Classification: Primary 60K15

Secondary 60E 10

\section{Introduction}

Markov chains, semi-Markov processes, and Markov additive processes are the stochastic models most widely used in applications. Reward functions on such processes are of great practical importance. We consider general reward functions on sample paths over deterministic time intervals for semi-Markov and Markov additive processes. These involve rewards associated with state transitions in their embedded Markov chains and linear rewards associated with state sojourns. In the case of Markov additive processes, the reward functions also involve linear rewards associated with state-indexed additive components. In particular, our reward functions cover as special cases fundamental quantities such as (i) sojourns in subsets of states (also called occupation times), (ii) weighted sojourns on the set of states (also called integral functionals of a random process), and (iii) the number of visits to a subset of states, all during a fixed time interval. The asymptotic theory for reward functions on Markov processes is very well developed and is therefore not a subject of this paper. The exact distributions of reward functions are of interest here. More specifically, of interest are those exact results which lead to the effective computation of these distributions. Note that the availability of an explicit closedform expression for a Laplace transform of a function allows the effective computation of the values of that function. Effective algorithms for numerical inversion of Laplace transforms, including multidimensional ones, are available (see Choudhury et al. (1994) and Abate et al. (1998)).

Received 8 May 2006; revision received 18 July 2006.

* Postal address: School of Mathematics and Statistics, The University of Western Australia, Crawley, WA 6009, Australia. Email address: stefanov@maths.uwa.edu.au 
There is a general, unifying approach for finding explicit closed-form expressions for the Laplace transforms of distributions of rewards accumulated within certain passage times, including the passage time from one state to another, or the same, state of the underlying Markov chain. This approach follows from Stefanov's (1995) results on semi-Markov and Markov additive processes. More specifically, Stefanov (1995, Propositions 4.1 and 5.1) proved in particular that the random quantities involved in the reward functions are components of canonical statistics of general noncurved exponential families if the process is observed within a first passage time from one state to another, or the same, state of the underlying Markov chain. These results lead to an explicit closed-form expression for the joint Laplace transform of these quantities (canonical statistics), based on basic analytical properties of exponential families of distributions (see Barndorff-Nielsen (1978, p. 114)). Since both the first passage times between states as well as the rewards accumulated within these passage times are linear functions of these random quantities, we obtain an explicit closed-form expression for the joint Laplace transform of any such first passage time, together with the accumulated reward within that passage time. Stefanov (1995) assumed that the holding time distributions in the case of semiMarkov processes belong to exponential families. The exponential families of distributions cover most of the discrete and continuous distributions which are important from a practical point of view. However, this restriction on the holding time distributions may be dropped, and explicit closed-form solutions for the joint Laplace transforms of the aforementioned random quantities follow from a method discussed in Stefanov (2000). The latter method is introduced through a detailed solution of a related problem in the same reference.

The exact distribution theory for rewards accumulated within deterministic time intervals has not been developed satisfactorily. For example, even for continuous-time Markov chains, there is no satisfactory solution leading to the effective computation of cumulative probabilities for reward functions. The literature on the exact distribution theory for reward functions is wide ranging. We confine ourselves to mentioning some key early sources in the area. Note that all available results on exact distributions usually involve matrix exponentials and/or other quantities not easily computable. Very early results on special reward functions, for either semiMarkov processes or Markov chains, can be found in Pyke (1961), Jewell (1963), McLean and Neuts (1967), Darroch and Morris (1968), and Çinlar (1969). Integral functionals on Markov processes were treated by Puri (1971), (1972). Sumita and Masuda (1987), Masuda and Sumita (1987), (1991), and Masuda (1993) derived results on the Laplace transforms of general reward functions. Sericola (1990), (2000) provided results on the exact distributions of sojourns and occupation times for continuous-time Markov chains. More recently, Bladt et al. (2002) treated a general reward function for continuous-time Markov chains.

In this paper we provide very neat identities linking relevant joint Laplace transforms associated with reward functions accumulated within a deterministic time interval with joint Laplace transforms associated with the counterparts of these reward functions accumulated within passage times between the states of the underlying Markov chains. Explicit closed-form expressions for the latter joint Laplace transforms (associated with such passage times) are derivable using the approach discussed above. Also, our identities do not involve any unknown quantities for the models considered. For example, in the case of a semi-Markov process, these identities involve the Laplace transforms of the holding time distributions, which are known. In the case of Markov additive processes they involve relevant Laplace transforms associated with the one-dimensional distributions of their state-indexed additive components (Lévy processes). The latter have simple explicit expressions if the additive components belong to general exponential families of Lévy processes. It is worth mentioning that most Markov 
additive processes which are important from a practical point of view have additive components from such families.

Finally, our results may be viewed in the spirit of other available results in the theory of Markov processes which link Laplace transforms of transient characteristics with Laplace transforms of first passage time characteristics. A good source of earlier results on the use of transforms for analysis of Markov models is Howard (1971).

The paper is organized as follows. Markov renewal and semi-Markov processes are treated in Section 2. Despite the equivalence between Markov renewal and semi-Markov processes, the corresponding reward functions have to be treated individually because their time parameters are different. Markov additive processes are treated in Section 3. To the best of the author's knowledge, the exact distribution theory for general reward functions on such processes has not been treated in the literature. For simplicity of notation we often use the same symbol for corresponding quantities in different Markov models. This will not confuse the reader because each model is discussed in an individual section or subsection.

\section{Markov renewal and semi-Markov processes}

Consider a Markov renewal process $(X, T)=(X(n), T(n))_{n \geq 0}$, where $X$ is a finite-state, discrete-time Markov chain with state space $\{1,2, \ldots, m\}$ and $T$ is the so-called additive component. It is assumed that $0=T(0)<T(1)<\cdots$. The holding time in state $j$ will be identified by a random variable $v_{j}$; that is, the distribution of $v_{j}$ is equal to the conditional distribution of $T(n+1)-T(n)$ given that $X(n)=j$. We assume that the Markov chain $X$ is irreducible. Let $Y$ be the semi-Markov process associated with the Markov renewal process $(X, T)$, that is,

$$
Y(t)=X(n) \quad \text { if } T(n) \leq t<T(n+1), \quad t \in[0, \infty) .
$$

The following reward functions $A(t)$ are of interest.

(i) For the semi-Markov process $Y$,

$$
A(t)=\sum_{i, j=1}^{m} k_{i, j} N_{i, j}(t)+\sum_{i=1}^{m} r_{i} T_{i}(t),
$$

where $N_{i, j}(t)$ is the number of one-step transitions from state $i$ to state $j$ of the semi-Markov process $Y(t)$ up to time $t, T_{i}(t)$ is the sojourn time in state $i$ up to time $t$ (note that $\sum_{i=1}^{m} T_{i}(t)=t$ ), and the $k_{i, j}$ and $r_{i}$ are real numbers. Without loss of generality, we will assume that the $r_{i}$ are nonnegative numbers. More specifically, if we replace the $r_{i}$ above by $r_{i}-\min _{1 \leq j \leq m} r_{j}$, then the new reward function, say $Q(t)$, is related to $A(t)$ through the identity $Q(t)=$ $A(t)-t \min _{1 \leq j \leq m} r_{j}$. The cumulative probabilities of $Q(t)$ yield those of $A(t)$, and vice versa.

(ii) For the Markov renewal process $(X, T)$,

$$
A(n)=\sum_{i, j=1}^{m} k_{i, j} N_{i, j}(n)+\sum_{i=1}^{m} r_{i} T_{i}(n),
$$

where $N_{i, j}(n)$ is the number of one-step transitions from state $i$ to state $j$ of the Markov chain $X$ within $n$ steps, $T_{i}(n)$ is the component of $T(n)$ associated with the entries to state $i$ within $n$ steps (with $T(n)=\sum_{i=1}^{m} T_{i}(n)$ ), and the $k_{i, j}$ and $r_{i}$ are real numbers. Again, without loss of generality, the $r_{i}$ are assumed to be nonnegative numbers. 
Using the same notation $N_{i, j}$ and $T_{i}$ in both the Markov renewal case and the semi-Markov case will not confuse the reader, since an integer argument $n$ will be used in the Markov renewal case and a continuous argument $t$ will be used in the semi-Markov case.

Denote by $\mathcal{L}\left[f\left(x_{1}, \ldots, x_{d}\right)\right] \equiv \mathcal{L}[f]$ the Laplace transform of a function $f\left(x_{1}, \ldots, x_{d}\right)$ defined on $\mathbb{R}_{+}^{d}$, where $\mathbb{R}_{+}=[0, \infty)$, and by $\mathcal{L}\left[G\left(x_{1}, \ldots, x_{d}\right)\right] \equiv \mathcal{L}[G]$ the Laplace transform of a probability measure $G\left(x_{1}, \ldots, x_{d}\right)$ on $\mathbb{R}^{d}$, that is

$$
\begin{aligned}
& \mathcal{L}[f]\left(s_{1}, \ldots, s_{d}\right)=\int_{0}^{\infty} \cdots \int_{0}^{\infty} \exp \left(-\sum_{i=1}^{d} s_{i} x_{i}\right) f\left(x_{1}, \ldots, x_{d}\right) \mathrm{d} x_{1} \cdots \mathrm{d} x_{d}, \\
& \mathcal{L}[G]\left(s_{1}, \ldots, s_{d}\right)=\int_{\mathbb{R}^{d}} \exp \left(-\sum_{i=1}^{d} s_{i} x_{i}\right) \mathrm{d} G\left(x_{1}, \ldots, x_{d}\right) .
\end{aligned}
$$

Also, $\mathcal{L}[Z](s)=\mathrm{E}\left(\mathrm{e}^{-s Z}\right)$ will denote the Laplace transform of a random variable $Z$. The quantities of interest are the Laplace transforms of the cumulative distribution functions $F_{A(t)}(a)=$ $\mathrm{P}(A(t) \leq a)$ and $F_{A(n)}(a)=\mathrm{P}(A(n) \leq a)$, that is,

$$
\begin{aligned}
& \mathcal{L}\left[F_{A(t)}(a)\right]\left(s_{1}, s_{2}\right)=\int_{0}^{\infty} \int_{0}^{\infty} \mathrm{e}^{-s_{1} t-s_{2} a} F_{A(t)}(a) \mathrm{d} t \mathrm{~d} a, \\
& \mathcal{L}\left[F_{A(n)}(a)\right]\left(s_{1}, s_{2}\right)=\sum_{n=0}^{\infty} \int_{0}^{\infty} \mathrm{e}^{-s_{1} n-s_{2} a} F_{A(n)}(a) \mathrm{d} a .
\end{aligned}
$$

For the sake of brevity, $\mathrm{P}_{i}(C)$ will stand for the conditional probability of an event $C$ given that the initial state is $i$, that is, $\mathrm{P}_{i}(C)=\mathrm{P}(C \mid X(0)=i)=\mathrm{P}(C \mid Y(0)=i)$.

\subsection{The semi-Markov case}

We consider the semi-Markov case first.

Denote by $\tau_{i, j}$ the waiting time to move from state $i$ to state $j$ in the discrete-time Markov chain $X$. If $i=j$ then $\tau_{j, j}$ (which is greater than 0 ) denotes the first return time to state $j$. Thus, $T\left(\tau_{i, j}\right)$ is the waiting time for the semi-Markov process $Y$ to move from state $i$ to state $j$. Denote by $A\left(T\left(\tau_{i, j}\right)\right)$ the accumulated reward within that waiting time and by $\mu_{T, A}\left(\tau_{i, j}\right)$ the joint probability measure of $T\left(\tau_{i, j}\right)$ and $A\left(T\left(\tau_{i, j}\right)\right)$. Recall that an explicit closed-form expression for the Laplace transform, $\mathcal{L}\left[\mu_{T, A}\left(\tau_{i, j}\right)\right]$, of the joint probability measure $\mu_{T, A}\left(\tau_{i, j}\right)$ is derivable for each semi-Markov model (see Section 1).

Theorem 2.1. Let $Y$ be the semi-Markov process introduced at the beginning of Section 2 and let $A(t)$ be the reward function introduced in (2.1). The following expression, where $p_{i}=\mathrm{P}(Y(0)=i)$, holds for the Laplace transform of $F_{A(t)}(a)$ in terms of the Laplace transforms of the joint probability measures $\mu_{T, A}\left(\tau_{i, j}\right)$ and the Laplace transforms of the holding times $v_{j}$ :

$$
\begin{aligned}
\mathscr{L}\left[F_{A(t)}(a)\right]\left(s_{1}, s_{2}\right)= & \sum_{i=1}^{m} p_{i} \sum_{j=1, j \neq i}^{m} \frac{\left(1-\mathcal{L}\left[v_{j}\right]\left(s_{1}+s_{2} r_{j}\right)\right) \mathcal{L}\left[\mu_{T, A}\left(\tau_{i, j}\right)\right]\left(s_{1}, s_{2}\right)}{s_{2}\left(s_{1}+s_{2} r_{j}\right)\left(1-\mathcal{L}\left[\mu_{T, A}\left(\tau_{j, j}\right)\right]\left(s_{1}, s_{2}\right)\right)} \\
& +\sum_{j=1}^{m} p_{j} \frac{1-\mathcal{L}\left[v_{j}\right]\left(s_{1}+s_{2} r_{j}\right)}{s_{2}\left(s_{1}+s_{2} r_{j}\right)\left(1-\mathcal{L}\left[\mu_{T, A}\left(\tau_{j, j}\right)\right]\left(s_{1}, s_{2}\right)\right)}
\end{aligned}
$$


Proof. For the cumulative distribution function of $A(t)$ we have

$$
F_{A(t)}(a)=\sum_{i, j=1}^{m} \mathrm{P}_{i}(A(t) \leq a, Y(t)=j) \mathrm{P}(Y(0)=i) .
$$

Let $\tau_{j}^{(k)}$ be the time of the $k$ th entry into state $j$ of the discrete-time Markov chain $X$ with $\tau_{j}^{(0)} \stackrel{j}{=} 0$, that is,

$$
\tau_{j}^{(k)}=\inf \left\{n>\tau_{j}^{(k-1)}: X(n)=j\right\} .
$$

Of course, $T\left(\tau_{j}^{(k)}\right)$ is the time of the $k$ th entry into state $j$ of the semi-Markov process $Y$. Hence,

$$
F_{A(t)}(a)=\sum_{k=0}^{\infty} \mathrm{P}_{i}\left(A(t) \leq a, T\left(\tau_{j}^{(k)}\right) \leq t<T\left(\tau_{j}^{(k+1)}\right), Y(t)=j\right) \mathrm{P}(Y(0)=i) .
$$

For the sake of brevity, we write

$$
b_{i, j}^{(k)}(t, a)=\mathrm{P}_{i}\left(A(t) \leq a, T\left(\tau_{j}^{(k)}\right) \leq t<T\left(\tau_{j}^{(k+1)}\right), Y(t)=j\right),
$$

and note that

$$
b_{i, j}^{(k)}(t, a)=\mathrm{P}_{i}\left(A\left(T\left(\tau_{j}^{(k)}\right)\right) \leq a-r_{j}\left(t-T\left(\tau_{j}^{(k)}\right)\right), T\left(\tau_{j}^{(k)}\right) \leq t<T\left(\tau_{j}^{(k+1)}\right), Y(t)=j\right)
$$

since

$$
A(t)=A\left(T\left(\tau_{j}^{(k)}\right)\right)+r_{j}\left(t-T\left(\tau_{j}^{(k)}\right)\right)
$$

if $Y(s)=j$ for $s \in\left[T\left(\tau_{j}^{(k)}\right), t\right]$. Furthermore, denoting by $v_{j}^{(k)}$ the holding time in state $j$ at the $k$ th visit to that state by the semi-Markov process $Y$, we note that, for $k \geq 1$,

$$
b_{i, j}^{(k)}(t, a)=\mathrm{P}_{i}\left(A\left(T\left(\tau_{j}^{(k)}\right)\right) \leq a-r_{j}\left(t-T\left(\tau_{j}^{(k)}\right)\right), T\left(\tau_{j}^{(k)}\right) \leq t, v_{j}^{(k)}>t-T\left(\tau_{j}^{(k)}\right)\right)
$$

since $\left\{T\left(\tau_{j}^{(k)}\right) \leq t, v_{j}^{(k)}>t-T\left(\tau_{j}^{(k)}\right)\right\}=\left\{T\left(\tau_{j}^{(k)}\right) \leq t<T\left(\tau_{j}^{(k+1)}\right), Y(t)=j\right\}$. For $k=0$, we have

$$
b_{i, j}^{(0)}(t, a)=\mathrm{P}_{i}\left(A(T(0)) \leq a-r_{i} t, T\left(\tau_{j}^{(1)}\right)>t, Y(t)=j\right),
$$

that is,

$$
b_{i, j}^{(0)}(t, a)= \begin{cases}\mathrm{P}\left(v_{j}>t\right) \mathbf{1}_{[0, \infty)}\left(a-r_{j} t\right) & \text { if } i=j, \\ 0 & \text { otherwise, }\end{cases}
$$

where $\mathbf{1}_{[0, \infty)}(\cdot)$ is the indicator function of the set $[0, \infty)$. Conditioning on

$$
\left(T\left(\tau_{j}^{(k)}\right), A\left(T\left(\tau_{j}^{(k)}\right)\right)\right),
$$

and bearing in mind that $v_{j}^{(k)}$ is independent of $\left(T\left(\tau_{j}^{(k)}\right), A\left(T\left(\tau_{j}^{(k)}\right)\right)\right)$, for $k \geq 1$ we obtain

$$
\begin{aligned}
b_{i, j}^{(k)}(t, a)=\int & \mathrm{P}\left(v_{j}^{(k)}>t-u_{1}\right) \mathbf{1}_{[0, \infty)}\left(t-u_{1}\right) \mathbf{1}_{[0, \infty)}\left(a-u_{2}-r_{j}\left(t-u_{1}\right)\right) \\
& \times \mathrm{d} \mu_{T\left(\tau_{j}^{(k)}\right), A\left(T\left(\tau_{j}^{(k)}\right)\right)}\left(u_{1}, u_{2}\right),
\end{aligned}
$$


where $\mu_{T\left(\tau_{j}^{(k)}\right), A\left(T\left(\tau_{j}^{(k)}\right)\right)}$ is the joint probability distribution of $T\left(\tau_{j}^{(k)}\right)$ and $A\left(T\left(\tau_{j}^{(k)}\right)\right)$. Letting

$$
\varphi_{j}(x, y)=\mathrm{P}\left(v_{j}>x\right) \mathbf{1}_{[0, \infty)}(x) \mathbf{1}_{[0, \infty)}\left(y-r_{j} x\right), \quad x, y \in \mathbb{R}_{+},
$$

we note that (2.5) can be written as

$$
b_{i, j}^{(k)}(t, a)=\int \varphi_{j}\left(t-u_{1}, a-u_{2}\right) \mathrm{d} \mu_{T\left(\tau_{j}^{(k)}\right), A\left(T\left(\tau_{j}^{(k)}\right)\right)}\left(u_{1}, u_{2}\right),
$$

that is, for $k \geq 1, b_{i, j}^{(k)}(t, a)$ is equal to the convolution of $\varphi_{j}(t, a)$ and the probability measure $\mu_{T\left(\tau_{j}^{(k)}\right), A\left(T\left(\tau_{j}^{k)}\right)\right)}$. Therefore, for the Laplace transform,

$$
\mathcal{L}\left[b_{i, j}^{(k)}\right]\left(s_{1}, s_{2}\right)=\int_{0}^{\infty} \int_{0}^{\infty} \mathrm{e}^{-s_{1} t-s_{2} a} b_{i, j}^{(k)}(t, a) \mathrm{d} t \mathrm{~d} a,
$$

of $b_{i, j}^{(k)}(t, a)$ we obtain the following identity:

$$
\mathcal{L}\left[b_{i, j}^{(k)}\right]\left(s_{1}, s_{2}\right)=\mathcal{L}\left[\varphi_{j}\right]\left(s_{1}, s_{2}\right) \mathcal{L}\left[\mu_{T\left(\tau_{j}^{(k)}\right), A\left(T\left(\tau_{j}^{(k)}\right)\right)}\right]\left(s_{1}, s_{2}\right) .
$$

It is a straightforward exercise to show that

$$
\mathcal{L}\left[\varphi_{j}(x, y)\right]\left(s_{1}, s_{2}\right)=\frac{\mathcal{L}\left[1-F_{v_{j}}\right]\left(s_{1}+s_{2} r_{j}\right)}{s_{2}},
$$

where $F_{v_{j}}$ is the cumulative distribution function of $v_{j}$, the holding time in state $j$. From basic operational properties of Laplace transforms (see Brychkov et al. (1992, p. 98)) we find that the Laplace transform, $\mathcal{L}\left[1-F_{v_{j}}\right](s)$, of $1-F_{v_{j}}$ is equal to $\left(1-\mathcal{L}\left[v_{j}\right](s)\right) / s$, where $\mathcal{L}\left[v_{j}\right]$ is the Laplace transform of the holding time $v_{j}$, that is, $\mathcal{L}\left[v_{j}\right]=\int_{0}^{\infty} \mathrm{e}^{-s t} \mathrm{~d} F_{v_{j}}(t)$. Thus,

$$
\mathcal{L}\left[\varphi_{j}\right]\left(s_{1}, s_{2}\right)=\frac{1-\mathcal{L}\left[v_{j}\right]\left(s_{1}+s_{2} r_{j}\right)}{s_{2}\left(s_{1}+s_{2} r_{j}\right)} .
$$

Since the consecutive entry times to a fixed state form a sequence of regeneration times, for $k \geq 1$ we obtain

$$
\mathcal{L}\left[\mu_{T\left(\tau_{j}^{(k)}\right), A\left(T\left(\tau_{j}^{(k)}\right)\right)}\right]\left(s_{1}, s_{2}\right)=\left(\mathcal{L}\left[\mu_{T, A}\left(\tau_{j, j}\right)\right]\left(s_{1}, s_{2}\right)\right)^{k}
$$

if the initial state is $j$, and

$$
\mathcal{L}\left[\mu_{T\left(\tau_{j}^{(k)}\right), A\left(T\left(\tau_{j}^{(k)}\right)\right)}\right]\left(s_{1}, s_{2}\right)=\mathcal{L}\left[\mu_{T, A}\left(\tau_{i, j}\right)\right]\left(s_{1}, s_{2}\right)\left(\mathcal{L}\left[\mu_{T, A}\left(\tau_{j, j}\right)\right]\left(s_{1}, s_{2}\right)\right)^{k-1}
$$

if the initial state is $i$. Recall that $\tau_{i, j}$ is the first passage time from state $i$ to state $j$ in the discrete-time Markov chain $X$. (Also recall the definition of $\mu_{T, A}\left(\tau_{i, j}\right)$ given prior to the statement of Theorem 2.1.) 
Therefore (see (2.4) and recall the expression for $b_{i, j}^{(0)}(t, a)$ ), we obtain the following expression for the Laplace transform of $F_{A(t)}(a)$ :

$$
\begin{aligned}
\mathscr{L}\left[F_{A(t)}(a)\right]= & \sum_{i, j=1}^{m} \sum_{k=0}^{\infty} \mathcal{L}\left[b_{i, j}^{(k)}(t, a)\right] \mathrm{P}(Y(0)=i) \\
= & \sum_{i, j=1, i \neq j}^{m} \sum_{k=1}^{\infty} \mathcal{L}\left[\varphi_{j}\right] \mathcal{L}\left[\mu_{T, A}\left(\tau_{i, j}\right)\right]\left(\mathcal{L}\left[\mu_{T, A}\left(\tau_{j, j}\right)\right]\right)^{k-1} \mathrm{P}(Y(0)=i) \\
& +\sum_{j=1}^{m} \sum_{k=0}^{\infty} \mathcal{L}\left[\varphi_{j}\right]\left(\mathcal{L}\left[\mu_{T, A}\left(\tau_{j, j}\right)\right]\right)^{k} \mathrm{P}(Y(0)=j) \\
= & \sum_{i, j=1, i \neq j}^{m} \frac{\mathcal{L}\left[\varphi_{j}\right] \mathcal{L}\left[\mu_{T, A}\left(\tau_{i, j}\right)\right]}{1-\mathcal{L}\left[\mu_{T, A}\left(\tau_{j, j}\right)\right]} \mathrm{P}(Y(0)=i) \\
& +\sum_{j=1}^{m} \frac{\mathcal{L}\left[\varphi_{j}\right]}{1-\mathcal{L}\left[\mu_{T, A}\left(\tau_{j, j}\right)\right]} \mathrm{P}(Y(0)=j) .
\end{aligned}
$$

Equation (2.3) is then derived by substituting into (2.7) the expression for $\mathcal{L}\left[\varphi_{j}\right]$ found in (2.6). The proof of Theorem 2.1 is thus complete.

In particular, from Theorem 2.1 we obtain the following corollary for the case of a continuoustime Markov chain, that is, if the holding times at states are exponentially distributed with holding time parameters $\lambda_{j}$; recall that, in this case, $\mathcal{L}\left[v_{j}\right](s)=\lambda_{j} /\left(\lambda_{j}+s\right)$.

Corollary 2.1. The following identity holds if the underlying model (the semi-Markov process $Y$ ) is a finite-state, continuous-time Markov chain:

$$
\begin{aligned}
\mathcal{L}\left[F_{A(t)}(a)\right]\left(s_{1}, s_{2}\right)= & \sum_{i=1}^{m} p_{i} \sum_{j=1, j \neq i}^{m} \frac{\mathcal{L}\left[\mu_{T, A}\left(\tau_{i, j}\right)\right]\left(s_{1}, s_{2}\right)}{s_{2}\left(\lambda_{j}+s_{1}+s_{2} r_{j}\right)\left(1-\mathcal{L}\left[\mu_{T, A}\left(\tau_{j, j}\right)\right]\left(s_{1}, s_{2}\right)\right)} \\
& +\sum_{j=1}^{m} p_{j} \frac{1}{s_{2}\left(\lambda_{j}+s_{1}+s_{2} r_{j}\right)\left(1-\mathcal{L}\left[\mu_{T, A}\left(\tau_{j, j}\right)\right]\left(s_{1}, s_{2}\right)\right)} .
\end{aligned}
$$

Here $p_{i}=\mathrm{P}(Y(0)=i)$ and $\mu_{T, A}\left(\tau_{i, j}\right)$ is the joint probability measure of the waiting time for the continuous-time Markov chain $Y$ to move from state $i$ to state $j$ and the accumulated reward within that waiting time.

Remark 2.1. (Discrete-time semi-Markov process.) Note that the statement of Theorem 2.1 also holds if the semi-Markov process considered has a discrete time parameter. That is, the holding time distributions are discrete on the set of the nonnegative integers. To see this, just replace the above integrals with respect to the time parameter with corresponding sums, and notice that all the expressions derived above also apply in this case.

\subsection{The Markov renewal case}

Now consider the cumulative distribution function of $A(n)$ for the Markov renewal case (ii). We have

$$
F_{A(n)}(a)=\mathrm{P}(A(n) \leq a)=\sum_{i, j=1}^{m} \mathrm{P}_{i}(A(n) \leq a, X(n)=j) \mathrm{P}(X(0)=i)
$$


and, for each $j$,

$$
\mathrm{P}_{j}(A(n) \leq a, X(n)=j)=\sum_{i=0}^{\infty} \mathrm{P}_{j}\left(A(n) \leq a, \tau_{j}^{(i)} \leq n<\tau_{j}^{(i+1)}, X(n)=j\right),
$$

where $\tau_{j}^{(i)}$ is again the $i$ th entry time into state $j$ by the discrete-time Markov chain $X$. In analogy with the case considered in Subsection 2.1, we write

$$
b_{j}^{(i)}(n, a)=\mathrm{P}_{j}\left(A(n) \leq a, \tau_{j}^{(i)} \leq n<\tau_{j}^{(i+1)}, X(n)=j\right) .
$$

Note that

$$
\begin{aligned}
b_{j}^{(i)}(n, a) & =\mathrm{P}_{j}\left(A\left(\tau_{j}^{(i)}\right) \leq a-r_{j}\left(n-\tau_{j}^{(i)}\right), \tau_{j}^{(i)} \leq n<\tau_{j}^{(i+1)}, X(n)=j\right) \\
& =\mathrm{P}_{j}\left(A\left(\tau_{j}^{(i)}\right) \leq a-r_{j}\left(n-\tau_{j}^{(i)}\right), \tau_{j}^{(i)} \leq n, h_{j}^{(i)}>n-\tau_{j}^{(i)}\right),
\end{aligned}
$$

where $h_{j}^{(i)}$ is the time spent in state $j$ at the $i$ th visit to that state by the discrete-time Markov chain $X$. Conditioning on $\left(\tau_{j}^{(i)}, A\left(\tau_{j}^{(i)}\right)\right)$, we obtain

$b_{j}^{(i)}(n, a)=\int \mathrm{P}\left(h_{j}^{(i)}>n-u_{1}\right) \mathbf{1}_{[0, \infty)}\left(n-u_{1}\right) \mathbf{1}_{[0, \infty)}\left(a-u_{2}-r_{j}\left(n-u_{1}\right)\right) \mathrm{d} \mu_{\tau_{j}^{(i)}, A\left(\tau_{j}^{(i)}\right)}\left(u_{1}, u_{2}\right)$,

where $\mu_{\tau_{j}^{(i)}, A\left(\tau_{j}^{(i)}\right)}$ is the joint probability distribution of $\tau_{j}^{(i)}$ and $A\left(\tau_{j}^{(i)}\right)$. In further analogy with the preceding case, we write

$$
\varphi_{j}(n, y)=\mathrm{P}\left(h_{j}^{(i)}>n\right) \mathbf{1}_{[0, \infty)}\left(y-r_{j} n\right), \quad n=0,1, \ldots, y \in \mathbb{R}_{+},
$$

and note that

$$
b_{j}^{(i)}(n, a)=\int \varphi_{j}\left(n-u_{1}, a-u_{2}\right) \mathrm{d} \mu_{\tau_{j}^{(i)}, A\left(\tau_{j}^{(i)}\right)}\left(u_{1}, u_{2}\right) .
$$

For a function $f(n, y), n \in\{0,1, \ldots\}, y \in \mathbb{R}_{+}$, the Laplace transform is

$$
\mathcal{L}[f]\left(s_{1}, s_{2}\right)=\sum_{n=0}^{\infty} \int_{0}^{\infty} \mathrm{e}^{-s_{1} n-s_{2} y} f(n, y) \mathrm{d} y .
$$

In view of (2.8), for the Laplace transform $\mathcal{L}\left[b_{j}^{(i)}\right]=\sum_{n=0}^{\infty} \int_{0}^{\infty} \mathrm{e}^{-s_{1} n-s_{2} a} b_{j}^{(i)}(n, a) \mathrm{d} a$ we obtain

$$
\mathscr{L}\left[b_{j}^{(i)}\right]\left(s_{1}, s_{2}\right)=\mathscr{L}\left[\varphi_{j}\right]\left(s_{1}, s_{2}\right) \mathcal{L}\left[\mu_{\tau_{j}^{(i)}, A\left(\tau_{j}^{(i)}\right)}\right]\left(s_{1}, s_{2}\right) .
$$

It is a simple exercise to see that

$$
\mathcal{L}\left[\varphi_{j}\right]\left(s_{1}, s_{2}\right)=\frac{1}{s_{2}} \sum_{n=0}^{\infty} \mathrm{e}^{-\left(s_{1}+s_{2} r_{j}\right) n} \mathrm{P}\left(h_{j}>n\right)=\frac{1-\mathcal{L}\left[h_{j}\right]\left(s_{1}+s_{2} r_{j}\right)}{s_{2}\left(1-\mathrm{e}^{-\left(s_{1}+s_{2} r_{j}\right)}\right)},
$$

where $h_{j}$ is the time spent at state $j$, at a visit to it, by the discrete-time Markov chain $X$ and $\mathcal{L}\left[h_{j}\right](s)=\sum_{n=0}^{\infty} \mathrm{e}^{-s n} \mathrm{P}\left(h_{j}=n\right)$ is its Laplace transform. Noting that the distribution of $h_{j}$ is geometric with support $\{1,2, \ldots\}$ and probability of 'success' $1-p_{j, j}$, where $p_{j, j}$ is 
the one-step transition probability from state $j$ to itself in the discrete-time Markov chain $X$, from (2.9) we obtain

$$
\mathcal{L}\left[\varphi_{j}\right]\left(s_{1}, s_{2}\right)=\frac{1}{s_{2}\left(1-p_{j, j} \mathrm{e}^{-s_{1}-s_{2} r_{j}}\right)} .
$$

Therefore, in analogy with the preceding case and denoting by $\mu_{\tau_{i, j}, A\left(\tau_{i, j}\right)}$ the joint probability measure of $\tau_{i, j}$ and $A\left(\tau_{i, j}\right)$, we arrive at the following result.

Theorem 2.2. Let $(X, T)$ be the Markov renewal process introduced at the beginning of Section 2 and let $A(n)$ be the reward function given in (2.2). The following identity holds for the Laplace transform of $F_{A(n)}(a)$ :

$$
\begin{aligned}
\mathcal{L}\left[F_{A(n)}(a)\right]\left(s_{1}, s_{2}\right)= & \sum_{i=1}^{m} p_{i} \sum_{j=1, j \neq i}^{m} \frac{\mathcal{L}\left[\mu_{\tau_{i, j}, A\left(\tau_{i, j}\right)}\right]\left(s_{1}, s_{2}\right)}{s_{2}\left(1-p_{j, j} \mathrm{e}^{-s_{1}-s_{2} r_{j}}\right)\left(1-\mathcal{L}\left[\mu_{\tau_{j, j}, A\left(\tau_{j, j}\right)}\right]\left(s_{1}, s_{2}\right)\right)} \\
& +\sum_{j=1}^{m} p_{j} \frac{1}{s_{2}\left(1-p_{j, j} \mathrm{e}^{-s_{1}-s_{2} r_{j}}\right)\left(1-\mathcal{L}\left[\mu_{\tau_{j, j}, A\left(\tau_{j, j}\right)}\right]\left(s_{1}, s_{2}\right)\right)} .
\end{aligned}
$$

Here $p_{i}=\mathrm{P}(X(0)=i)$ and $\mu_{\tau_{i, j}, A\left(\tau_{i, j}\right)}$ is the joint probability measure of the waiting time for the discrete-time Markov chain $X$ to move from state $i$ to state $j$ and the accumulated reward within that waiting time.

Again recall that a general method for the derivation of explicit closed-form expressions for the Laplace transforms $\mathcal{L}\left[\mu_{\tau_{i, j} A\left(\tau_{i, j}\right)}\right]$ was discussed in Section 1 .

\section{Markov additive processes}

A Markov additive process is a two-dimensional Markov process $(Z(t), V(t))_{t \geq 0}(t$ is a continuous time parameter) on the state space $I \times \mathbb{R}(I=\{1,2, \ldots, m\})$, with the following properties.

(a) The increments $V\left(t_{1}\right)-V(0), V\left(t_{2}\right)-V\left(t_{1}\right), \ldots, V\left(t_{n}\right)-V\left(t_{n-1}\right)$, where $t_{1}<t_{2}<$ $\cdots<t_{n}$, are conditionally independent given $Z\left(t_{1}\right), \ldots, Z\left(t_{n}\right)$.

(b) The conditional distribution of $V\left(t_{k}\right)-V\left(t_{k-1}\right)$ given that both $Z\left(t_{k-1}\right)=i$ and $Z\left(t_{k}\right)=j$ depends only on $t_{k}-t_{k-1}, i$, and $j$.

We will assume that $V(0)=0$. The process $\{Z(t)\}_{t \geq 0}$ is a finite-state, continuous-time Markov process and the process $\{V(t)\}_{t \geq 0}$ is usually called the additive component of the Markov additive process $(Z, V)$. Let

$$
V_{j}(t)=\int_{0}^{t} \mathbf{1}_{\{Z(s)=j\}} \mathrm{d} V(s), \quad j=1,2, \ldots, m,
$$

where $\mathbf{1}_{\{Z(s)=j\}}$ is the indicator function of the event $\{Z(s)=j\}$. For an alternative and equivalent definition of the $V_{j}(t)$, see Stefanov (1995, p. 1088). Of course, $V(t)=\sum_{j=1}^{m} V_{j}(t)$. Also, the $V_{j}$ are Lévy processes and the additive component $V$ evolves as $V_{j}$ during sojourns of $Z$ at state $j$ (see Asmussen (2003, p. 310)). The finite-dimensional distributions of the Lévy process $V_{j}$ are determined by the distribution of the increment random variable $V_{j}(t)$. We will assume that the processes $V_{j}$ are nonnegative, that is, $0 \leq V(s) \leq V(t)$ if $s<t$. 
The reward function of interest has the following general form:

$$
A(t)=\sum_{i, j=1}^{m} k_{i, j} N_{i, j}(t)+\sum_{i=1}^{m} r_{i} S_{i}(t)+\sum_{i=1}^{m} v_{i} V_{i}(t)
$$

Here $N_{i, j}(t)$ is the number of one-step transitions from state $i$ to state $j$ of the Markov process $Z(t)$ up to time $t, S_{i}(t)$ is the sojourn time in state $i$ of the Markov process $Z(t)$ up to time $t$, the $v_{i}$ are nonnegative numbers, and the $k_{i, j}$ and $r_{i}$ are real numbers. As in the preceding cases we will assume, without loss of generality, that the $r_{i}$ are nonnegative numbers.

Let

$$
b_{i, j}^{(k)}(t, a)=\mathrm{P}_{i}\left(A(t) \leq a, \rho_{j}^{(k)} \leq t<\rho_{j}^{(k+1)}, Z(t)=j\right),
$$

where $\rho_{j}^{(k)}$ is the time of the $k$ th entry to state $j$ by the Markov process $Z(t)$, and $\mathrm{P}_{i}(\cdot)=$ $\mathrm{P}(\cdot \mid Z(0)=i)$. Note that

$$
\begin{aligned}
& b_{i, j}^{(k)}(t, a) \\
& \quad=\mathrm{P}_{i}\left(A\left(\rho_{j}^{(k)}\right) \leq a-r_{j}\left(t-\rho_{j}^{(k)}\right)-v_{j}\left(V_{j}(t)-V_{j}\left(\rho_{j}^{(k)}\right)\right), \rho_{j}^{(k)} \leq t<\rho_{j}^{(k+1)}, Z(t)=j\right)
\end{aligned}
$$

since $A(t)=\underset{(k)}{A}\left(\rho_{j}^{(k)}\right)+r_{j}\left(t-\rho_{j}^{(k)}\right)+v_{j}\left(V_{j}(t)-V_{j}\left(\rho_{j}^{(k)}\right)\right)$ if $Z(s)=j$ for $s \in\left[\rho_{j}^{(k)}, t\right]$. Also, denoting by $v_{j}^{(k)}$ the holding time in state $j$ at the $k$ th visit to that state by the continuous-time Markov chain $Z$, note that, for $k \geq 1$,

$b_{i, j}^{(k)}(t, a)=\mathrm{P}_{i}\left(A\left(\rho_{j}^{(k)}\right) \leq a-r_{j}\left(t-\rho_{j}^{(k)}\right)-v_{j}\left(V_{j}(t)-V_{j}\left(\rho_{j}^{(k)}\right)\right), \rho_{j}^{(k)} \leq t, v_{j}^{(k)}>t-\rho_{j}^{(k)}\right)$

since

$$
\left\{\rho_{j}^{(k)} \leq t, v_{j}^{(k)}>t-\rho_{j}^{(k)}\right\}=\left\{\rho_{j}^{(k)} \leq t<\rho_{j}^{(k+1)}, Z(t)=j\right\} .
$$

Now, by conditioning on $\left(\rho_{j}^{(k)}, A\left(\rho_{j}^{(k)}\right)\right)$, for $k \geq 1$ we obtain

$$
b_{i, j}^{(k)}(t, a)=\int H \mathbf{1}_{[0, \infty)}\left(t-u_{1}\right) \mathrm{d} \mu_{\rho_{j}^{(k)}, A\left(\rho_{j}^{(k)}\right)}\left(u_{1}, u_{2}\right),
$$

where

$H=\mathrm{P}\left(v_{j}^{(k)}>t-u_{1}, V_{j}(t)-V_{j}\left(\rho_{j}^{(k)}\right) \leq \frac{a-u_{2}-r_{j}\left(t-u_{1}\right)}{v_{j}} \mid \rho_{j}^{(k)}=u_{1}, A\left(\rho_{j}^{(k)}\right)=u_{2}\right)$

(we assume here that $v_{j}>0$; for the case where $v_{j}=0$, see the comment made prior to the statement of Theorem 3.1 below) and $\mu_{\rho_{j}^{(k)}, A\left(\rho_{j}^{(k)}\right)}$ is the joint probability distribution of $\rho_{j}^{(k)}$ and $A\left(\rho_{j}^{(k)}\right)$. Furthermore, note that

$$
H=\left(1-F_{v_{j}^{(k)}}\left(t-u_{1}\right)\right) F_{V_{j}\left(t-u_{1}\right)}\left(\frac{a-u_{2}-r_{j}\left(t-u_{1}\right)}{v_{j}}\right)
$$

(recall that by $F_{X}$ we denote the cumulative distribution function of a random variable $X$ ) because the conditional distribution of the increment $V_{j}(t)-V_{j}\left(\rho_{j}^{(k)}\right)$ given that $\rho_{j}^{(k)}=u_{1}$, and assuming that $v_{j}^{(k)}>t-\rho_{j}^{(k)}$ (that is, $Z(s)=j$ on the time interval $\left[\rho_{j}^{(k)}, t\right]$ ), is equal to the one-dimensional distribution of the Lévy process $V_{j}$ at time $t-u_{1}$, and $v_{j}(k)$ is independent of $\rho_{j}^{(k)}$ and $A\left(\rho_{j}^{(k)}\right)$. 
Therefore, in view of (3.1) and (3.2), we find that

$$
b_{i, j}^{(k)}(t, a)=\int \varphi_{j}\left(t-u_{1}, a-u_{2}\right) \mathrm{d} \mu_{\rho_{j}^{(k)}, A\left(\rho_{j}^{(k)}\right)}\left(u_{1}, u_{2}\right),
$$

where

$$
\varphi_{j}(t, a)=\left(1-F_{v_{j}^{(k)}}(t)\right) F_{V_{j}(t)}\left(\frac{a-r_{j} t}{v_{j}}\right), \quad t, a \in \mathbb{R}_{+} .
$$

Recalling that $v_{j}^{(k)}$ is exponentially distributed with parameter $\lambda_{j}$, since the process $Z$ is a continuous-time Markov chain, we obtain

$$
\varphi_{j}(t, a)=\mathrm{e}^{-\lambda_{j} t} F_{V_{j}(t)}\left(\frac{a-r_{j} t}{v_{j}}\right) .
$$

Thus,

$$
\mathcal{L}\left[b_{i, j}^{(k)}(t, a)\right]=\mathcal{L}\left[\varphi_{j}(t, a)\right] \mathcal{L}\left[\mu_{\rho_{j}^{(k)}, A\left(\rho_{j}^{(k)}\right)}\right] .
$$

Denote by $\rho_{i, j}$ the waiting time for the Markov chain $Z$ to move from state $i$ to state $j$. In analogy with the preceding cases, since the $\rho_{j}^{(k)}$ form a sequence of regeneration times, we find that

$$
\mathcal{L}\left[\mu_{\rho_{j}^{(k)}, A\left(\rho_{j}^{(k)}\right)}\right]= \begin{cases}\left(\mathcal{L}\left[\mu_{\rho_{j, j}, A}\right]\right)^{k} & \text { if } Z(0)=j, \\ \mathcal{L}\left[\mu_{\rho_{i, j}, A}\right]\left(\mathcal{L}\left[\mu_{\rho_{j, j}, A}\right]\right)^{k-1} & \text { if } Z(0)=i \neq j,\end{cases}
$$

where $\mu_{\rho_{i, j}, A}$ is the joint probability measure of $\rho_{i, j}$ and the reward accumulated within that waiting time.

It is not a difficult exercise to find the Laplace transform of $\varphi_{j}(t, a)$. More specifically, by applying basic operational properties of Laplace transforms in the case where the distribution of $V_{j}(t)$ is continuous, we obtain

$$
\begin{aligned}
\mathcal{L}\left[\varphi_{j}(t, a)\right]\left(s_{1}, s_{2}\right) & =\int_{0}^{\infty} \int_{0}^{\infty} \mathrm{e}^{-s_{1} t-s_{2} a} \mathrm{e}^{-\lambda_{j} t} F_{V_{j}(t)}\left(\frac{a-r_{j} t}{v_{j}}\right) \mathrm{d} a \mathrm{~d} t \\
& =\int_{0}^{\infty} \mathrm{e}^{-s_{1} t-s_{2} r_{j} t} \mathrm{e}^{-\lambda_{j} t} \mathcal{L}\left[F_{V_{j}(t)}\left(a / v_{j}\right)\right]\left(s_{2}\right) \mathrm{d} t \\
& =\int_{0}^{\infty} v_{j} \mathrm{e}^{-s_{1} t-s_{2} r_{j} t} \mathrm{e}^{-\lambda_{j} t} \mathcal{L}\left[F_{V_{j}(t)}(a)\right]\left(s_{2}\right) \mathrm{d} t \\
& =\int_{0}^{\infty} \frac{v_{j} \mathrm{e}^{-s_{1} t-s_{2} r_{j} t} \mathrm{e}^{-\lambda_{j} t}}{s_{2}} \mathcal{L}\left[V_{j}(t)\right]\left(s_{2}\right) \mathrm{d} t \\
& =\frac{v_{j} \mathcal{L}\left[\mathcal{L}\left[V_{j}(t)\right]\left(s_{2}\right)\right]\left(\lambda_{j}+s_{1}+s_{2} r_{j}\right)}{s_{2}},
\end{aligned}
$$

where $\mathcal{L}\left[V_{j}(t)\right]\left(s_{2}\right)$ is the Laplace transform of the random variable $V_{j}(t)$ and $\mathcal{L}\left[\mathcal{L}\left[V_{j}(t)\right]\left(s_{2}\right)\right]$ is the Laplace transform of $\mathcal{L}\left[V_{j}(t)\right]\left(s_{2}\right)$, the latter viewed as a function of $t$ with $s_{2}$ fixed. If the distribution of $V_{j}(t)$ is discrete and its state space is the set of nonnegative integers, we can easily recover the same expression as (3.6). Furthermore, it is easy to see that, in the case $k=0$, for the $b_{i, j}^{(k)}(t, a)$ we obtain

$$
b_{i, j}^{(0)}(t, a)= \begin{cases}\mathrm{e}^{-\lambda_{j} t} F_{V_{j}(t)}\left(\frac{a-r_{j} t}{v_{j}}\right) & \text { if } i=j, \\ 0 & \text { otherwise. }\end{cases}
$$


Note that $b_{i, j}^{(0)}(t, a)=\varphi_{j}(t, a)$ (see (3.3)). Therefore, in view of (3.4) and (3.5), for each $i$ and $j$ we have

$$
\mathcal{L}\left[b_{i, j}^{(k)}(t, a)\right]= \begin{cases}\mathcal{L}\left[\varphi_{j}(t, a)\right]\left(\mathcal{L}\left[\mu_{\rho_{j, j}, A}\right]\right)^{k}, & i=j, k \geq 0, \\ \mathcal{L}\left[\varphi_{j}(t, a)\right] \mathcal{L}\left[\mu_{\rho_{i, j}, A}\right]\left(\mathcal{L}\left[\mu_{\rho_{j, j}, A}\right]\right)^{k-1}, & i \neq j, k \geq 1 .\end{cases}
$$

At a certain stage we assumed that $v_{j}>0$. Note that, for $v_{j}=0$ this case reduces to that of a continuous-time Markov chain. Therefore, we arrive at the following theorem.

Theorem 3.1. Let $A(t)$ be the reward function introduced at the beginning of this section. For each $i$ and each $j$ such that $v_{j}>0$, the following identities hold:

$$
\begin{aligned}
\mathrm{P}_{i}(A(t) & \leq a, Z(t)=j) \\
= & \mathcal{L}^{-1}\left[\frac{v_{j} \mathcal{L}\left[\mathcal{L}\left[V_{j}(t)\right]\left(s_{2}\right)\right]\left(\lambda_{j}+s_{1}+s_{2} r_{j}\right) \mathcal{L}\left[\mu_{\rho_{i, j}, A}\right]\left(s_{1}, s_{2}\right)}{s_{2}\left(1-\mathcal{L}\left[\mu_{\rho_{j, j}, A}\right]\left(s_{1}, s_{2}\right)\right)}\right](t, a), \quad i \neq j, \\
\mathrm{P}_{j}(A(t) & \leq a, Z(t)=j) \\
= & \mathcal{L}^{-1}\left[\frac{v_{j} \mathcal{L}\left[\mathcal{L}\left[V_{j}(t)\right]\left(s_{2}\right)\right]\left(\lambda_{j}+s_{1}+s_{2} r_{j}\right)}{s_{2}\left(1-\mathcal{L}\left[\mu_{\rho_{j, j}, A}\right]\left(s_{1}, s_{2}\right)\right)}\right](t, a) .
\end{aligned}
$$

Here $\mathcal{L}^{-1}$ denotes the inverse Laplace transform, the Laplace transform $\mathcal{L}\left[\mathcal{L}\left[V_{j}(t)\right]\left(s_{2}\right)\right]$ is as introduced in (3.6), and, recall, $\mu_{\rho_{i, j}, \text { A }}$ denotes the joint distribution of the first waiting time $\rho_{i, j}$ and the accumulated reward within that waiting time. Furthermore, for each $i$ and each $j$ such that $v_{j}=0$, the following identities hold:

$$
\begin{aligned}
& \mathrm{P}_{i}(A(t) \leq a, Z(t)=j)=\mathcal{L}^{-1}\left[\frac{\mathcal{L}\left[\mu_{\rho_{i, j}, A}\right]\left(s_{1}, s_{2}\right)}{s_{2}\left(\lambda_{j}+s_{1}+s_{2} r_{j}\right)\left(1-\mathcal{L}\left[\mu_{\rho_{j, j}, A}\right]\left(s_{1}, s_{2}\right)\right)}\right](t, a), \\
& \mathrm{P}_{j}(A(t) \leq a, Z(t)=j)=\mathcal{L}^{-1}\left[\frac{1}{s_{2}\left(\lambda_{j}+s_{1}+s_{2} r_{j}\right)\left(1-\mathcal{L}\left[\mu_{\rho_{j, j}, A}\right]\left(s_{1}, s_{2}\right)\right)}\right](t, a) .
\end{aligned}
$$

Recall that a general method, based on Stefanov (1995), was discussed in Section 1 for the derivation of explicit closed-form expressions for the Laplace transforms $\mathcal{L}\left[\mu_{\rho_{i, j}, A}\right]$.

If, for each $j$, the Lévy process $V_{j}$ is a member of a natural exponential family of Lévy processes (for a formal definition of these families, see Küchler and Sørensen (1997, p. 7)), then the Laplace transform $\mathcal{L}\left[\mathcal{L}\left[V_{j}(t)\right]\left(s_{2}\right)\right](s)$ has an explicit simple expression. More specifically, let the process $V_{j}$ belong to such a family. That is, for each $t$, assume that the distribution of the random variable $V_{j}(t)$ has a density, with respect to a $\sigma$-finite measure, of the form

$$
f_{V_{j}(t)}(x)=\exp \left(\theta_{j} x-t k_{j}\left(\theta_{j}\right)\right),
$$

where $\theta_{j}$ is a parameter called the natural parameter of this exponential family of densities and $k_{j}\left(\theta_{j}\right)$ is usually called either a cumulant transform (Küchler and Sørensen $(1997$, p. 8)) or a $\log$ Laplace transform (Brown (1986, p. 1)). From the basic properties of exponential families, we have $\mathcal{L}\left[V_{j}(t)\right](s)=\exp \left(t\left[k_{j}\left(\theta_{j}-s\right)-k_{j}\left(\theta_{j}\right)\right]\right)$. Thus, we find that

$$
\begin{aligned}
\mathscr{L}\left[\mathcal{L}\left[V_{j}(t)\right]\left(s_{2}\right)\right]\left(\lambda_{j}+s_{1}+s_{2} r_{j}\right) & =\int_{0}^{\infty} \exp \left(-t\left[\lambda_{j}+s_{1}+s_{2} r_{j}+k_{j}\left(\theta_{j}\right)-k_{j}\left(\theta_{j}-s_{2}\right)\right]\right) \mathrm{d} t \\
& =\frac{1}{\lambda_{j}+s_{1}+s_{2} r_{j}+k_{j}\left(\theta_{j}\right)-k_{j}\left(\theta_{j}-s_{2}\right)} .
\end{aligned}
$$


For example, if the process $V_{j}$ is a Poisson process with a rate parameter $\lambda$, then the natural parameter is $\theta_{j}=\ln \lambda$ and the cumulant transform is given by $k_{j}\left(\theta_{j}\right)=\mathrm{e}^{\theta_{j}}-1$ (see Küchler and Sørensen (1997, p. 9)).

\section{References}

Abate, J., Choudhury, G. L. And Whitt, W. (1998). Numerical inversion of multidimensional Laplace transforms by the Laguerre method. Performance Eval. 31, 229-243.

Asmussen, S. (2003). Applied Probability and Queues, 2nd edn. Springer, New York.

BARndorfF-Nielsen, O. (1978). Information and Exponential Families. John Wiley, Chichester.

Bladt, M., Meini, B., Neuts, M. F. and Sericola, B. (2002). Distributions of reward functions on continuous-time Markov chains. In Matrix-Analytic Methods (Adelaide, 2002), World Scientific, River Edge, NJ, pp. 39-62.

Brown, L. (1986). Fundamentals of Statistical Exponential Families. IMS, Hayward, CA.

Brychkov, Y. A., Gleske, H. J., Prudnikov, A. P. And Tuan, V. K. (1992). Multidimensional Integral Transformations. Gordon and Breach, Philadelphia, PA.

Choudhury, G. L., Lucantoni, D. M. And Whitt, W. (1994). Multidimensional transform inversion with applications to the transient M/G/1 queue. Ann. Appl. Prob. 4, 719-740.

Çinlar, E. (1969). Markov renewal theory. Adv. Appl. Prob. 1, 123-187.

Darroch, J. N. AND Morris, K. W. (1968). Passage-time generating functions for continuous-time finite Markov chains. J. Appl. Prob. 5, 414-426.

Howard, R. A. (1971). Dynamic Probabilistic Systems, Vols I and II. John Wiley, New York.

JEWELL, W. S. (1963). Markov renewal programming. I. Formulation, finite return models. Operat. Res. 11, $938-948$.

KüChler, U. AND Sørensen, M. (1997). Exponential Families of Stochastic Processes. Springer, New York.

Masuda, Y. (1993). Partially observable semi-Markov reward processes. J. Appl. Prob. 30, 548-560.

Masuda, Y. ANd Sumita, U. (1987). Analysis of a counting process associated with a semi-Markov process: number of entries into a subset of state space. Adv. Appl. Prob. 19, 767-783.

Masuda, Y. ANd Sumita, U. (1991). A multivariate reward process defined on a semi-Markov process and its firstpassage-time distributions. J. Appl. Prob. 28, 360-373.

McLean, R. A. And Neuts, M. F. (1967). The integral of a step function defined on a semi-Markov process. SIAM J. Appl. Math. 15, 726-737.

PURI, P. S. (1971). A method for studying the integral functionals of stochastic processes with applications. I. Markov chain case. J. Appl. Prob. 8, 331-343.

PURI, P. S. (1972). A method for studying the integral functionals of stochastic processes with applications. II. Sojourn time distributions for Markov chains. Z. Wahrscheinlichkeitsth. 23, 85-96.

PyKe, R. (1961). Markov renewal processes with finitely many states. Ann. Math. Statist. 32, 1243-1259.

Sericola, B. (1990). Closed-form solution for the distribution of the total time spent in a subset of states of a homogeneous Markov process during a finite observation period. J. Appl. Prob. 27, 713-719.

Sericola, B. (2000). Occupation times in Markov processes. Stoch. Models 16, 479-510.

STEFanov, V. T. (1995). Explicit limit results for minimal sufficient statistics and maximum likelihood estimators for some Markov processes: exponential families approach. Ann. Statist. 23, 1073-1101.

Stefanov, V. T. (2000). On some waiting time problems. J. Appl. Prob. 37, 756-764.

Sumita, U. AND MASUdA, Y. (1987). An alternative approach to the analysis of finite semi-Markov and related processes. Stoch. Models 3, 67-87. 